\title{
Peripheral Vestibular Function in Patients with Fibromyalgia
}

\author{
Aya S. Mohamed ${ }^{1 *}$, Alaa A. Abousetta', Mohamed A. Hefny' ${ }^{2}$, and \\ Alaa M. Farid ${ }^{3}$ \\ ${ }^{1}$ Audio-Vestibular Unit, Department of Otolaryngology, Faculty of Medicine, Suez Canal University, ${ }^{2}$ Department \\ of Physical Medicine, Rheumatology and Rehabilitation, Faculty of Medicine, Suez Canal University, and Depart- \\ ment of Otolaryngology, Faculty of Medicine, Suez Canal University, Egypt.
}

\begin{abstract}
Background: Fibromyalgia (FM) is a non-inflammatory musculoskeletal chronic syndrome, whose etiology is unknown, characterized by diffuse pain, many patients with FM were reported to have dizziness which is attributed to hypotension and the autonomic nervous system (ANS) dysfunction. Aim: To improve management of patients with fibromyalgia having dizziness. Objective: to assess peripheral vestibular functions in patients with fibromyalgia. Subjects and Methods: After obtaining approval from the Ethics Committee of the Suez Canal University Hospital (registration number:3646) and written informed patient consent, a Cross-sectional study was conducted at Suez Canal University hospital from June 2018 to December 2019 in the Audio-Vestibular Clinic of the E.N.T department, including 30 female subjects whose age ranged from 22 to 50 years and were divided into Study group that comprised patients diagnosed with fibromyalgia and Control group rather healthy individuals. The vestibular assessment was done using pure tone audiometry, Bedside tests, and video-nystagmography (VNG). Results: There was no statistically significant difference between both groups regarding results of bedside examinations and VNG results as a caloric test ( $p$-value $=0.6$ ) however, there was a trend towards positive results and an increase in vestibular weakness in the fibromyalgia group compared to the control group. Conclusion: Fibromyalgia is unlikely to be a cause of peripheral vestibular dysfunction, so vestibular testing is not routinely recommended for such patients.
\end{abstract}

Keywords: Fibromyalgia, Peripheral vestibular system, Suez Canal University Hospitals, Egypt.

\section{Introduction}

Fibromyalgia (FM) is a non-inflammatory musculoskeletal chronic syndrome, whose etiology is unknown, characterized by a diffuse pain, increase in palpation sensitivity and such symptoms as tiredness, insomnia, anxiety, depression, cold intolerance and otologic complaints. Although the FM-controlling physiological mechanisms have not been determined, neuroendocrine, gene- tic or molecular factors can be involved ${ }^{(1)}$. Many patients with FM were reported to have dizziness which is attributed to hypotension and autonomic nervous system (ANS) dysfunction and in this study we want to explore if dizziness and imbalance occur due to these factors only or due to a direct effect of fibromyalgia on the vestibular system ${ }^{(2)}$. Peripheral vestibular function can be assessed by Bedside vestibular assessment that include 1) Spontaneous

\footnotetext{
*Corresponding Author: ayasaber1991@yahoo.com
} 
Nystagmus and Other Involuntary Eye Movements (In unilateral peripheral differentiation, the spontaneous nystagmus is mixed horizontal-torsional beating away from the lesion side(3)). 2) Gaze-evoked nystagmus (GEN is one of the most sensitive ocular motor signs for central pathologies in patients with acute vestibular syndrome $\left.{ }^{(4)}\right)$. 3) Head-shaking nystagmus (In unilateral peripheral vestibulopathy, the typical pattern of HSN initially consists of contra-lesional nystagmus. Central patterns of HSN include unusually strong HSN elicited by weak head-shaking, intense HSN in patients without caloric paresis, ipsi-lesional $\mathrm{HSN}$, and its direction vertical or torsional nystagmus developing in response to horizontal head-shaking $\left.{ }^{(5)}\right)$. 4) The Dix-Hallpike maneuver (It is the gold-standard test for a diagnosis of BPPV (benign paroxysmal positional nystagmus), involving the posterior semicircular canal (PC-BPPV). In PCBPPV the elicited nystagmus would be mixed upbeat and torsional( ${ }^{(6)}$. The $\mathrm{HC}$ can be maximally stimulated using the supine roll test. Two types of nystagmus may be observed in BPPV involving the $\mathrm{HC}$ (HCBPPV): geotropic nystagmus beating toward the ground (lower ear) or apogeotropic nystagmus beating toward the ceiling $^{(6)}$. In the rare subtype of BPPV involving the anterior semicircular canal (AC-BPPV), elicit downbeat nystagmus $(7)$. And 5) Stepping test (The stepping test is useful for detecting vestibular deficits by disrupting proprioceptive compensation(6). Peripheral vestibular function can also be assessed by Caloric irrigation [Bithermal caloric testing is the most used method to evoke vestibular nystagmus. Warm and cool water (or air) irrigations are applied to each ear $\left.{ }^{(8)}\right]$.

\section{Subjects and Methods}

The study was conducted at Suez Canal University hospital from June 2018 to
December 2019 in Audio-Vestibular Clinic of the E.N.T department. The target population were all female subjects whose age ranged from 22 to 50 years and were divided into; study group that comprised patients diagnosed with fibromyalgia according to ACR diagnostic criteria and patients were taken from the Rheumatology outpatient clinic and control group that consists of rather healthy individuals. Inclusion criteria included female patients diagnosed with fibromyalgia and patient aged between 22 to 50 years old. Exclusion criteria included patients with previous history of peripheral vestibular weakness as result of vestibular neuritis, BPPV, Meniere's disease, CNS disorders and patients with postural hypotension. Study design was cross sectional comparative study.

\section{Methods}

After obtaining approval from the Ethics Committee of the Suez Canal University Hospital (registration number:3646), and written informed patient consent with an explanation regarding the purpose, effects, technique of the tests that were applied in both ( patient with fibromyalgia that diagnosed by clinical examination to tender pointslareas according to the ACR diagnostic criteria that performed at the Rheumatology clinic by a trained physicians, then patients were referred to the audio-vestibular clinic) and control group , The vestibular assessment was performed in both groups as follows: 1 - Clinical history for the presence of previous episodes of rotational vertigo and sensation of unsteadiness or imbalance. 2- Pure tone audiometry for frequencies between $250 \mathrm{~Hz}$ and $8000 \mathrm{~Hz}$; the exam was performed in a quiet room with a half octave precision. 3Speech audiometry was performed by doing Speech Recognition Threshold (SRT) and the materials that were used in speech threshold testing were spondees, (two-syllable words) by using live voice materials. 
Speech discrimination score (SDS) was also assessed and the most common materials for (SDS) were the monosyllabic words by using the open set protocol and SDS was defined as the percentage of words correctly identified(9). 4- Evaluation of vestibular system function was performed by $A$ ) Bedside tests that included the following:

1- Spontaneous Nystagmus and Other Involuntary Eye Movements.

2- Gaze-evoked nystagmus: It was done by asking the patient to follow doctor's finger in horizontal and vertical planes.

3- Head-shaking nystagmus: Head-shaking nystagmus (HSN) was assessed using either a passive (by the examiner) or active (by the patient) head-shaking maneuver. The patient's head was pitched forward by approximately $20^{\circ}$ to bring the horizontal semicircular canals ( $\mathrm{HCs}$ ) into the plane of stimulation, and then the head was shaken horizontally in a sinusoidal fashion at a rate of about $2-3 \mathrm{~Hz}$ with amplitude of $20^{\circ}$ for 15 seconds.

4- The Dix-Hallpike maneuver: In examination of A.C and P.C while seated on the examination table, the patient's head was turned $45^{\circ}$ toward the side to be tested, then was moved to a supine position, ending with the head hanging $20^{\circ}$ below the examination table. In examination of H.C using the supine roll test, in which the patient's head was first flexed forward about $30^{\circ}$ align the $\mathrm{HC}$ with the earth vertical, and then turned about $90^{\circ}$ to each side.

5- Stepping test: The examiner asked the patient to march in a fixed position with the arms extended and eyes closed.

B) Videonystagmography (VNG):

It was done to exclude peripheral vestibular lesion and detect any oculomotor tests or gaze abnormalities, it includes:

\footnotetext{
- Spontaneous nystagmus.

- Gaze evoked nystagmus.

- Oculomotor testing (saccade, smooth pursuit, and optokinetic tests).

- Positional testing
}

- Dix-Hallpike (Dynamic positioning).

- Caloric test to exclude peripheral vestibulopathy.

Water irrigations were applied to each ear and the maximum velocity of the slow component of nystagmus from each ear was determined.

\section{Statistical Analysis}

The statistical analysis was performed using IBM SPSS Statistics ${ }^{\circledR} 22$ for Windows 8 operating system .Descriptive data was expressed as median and interquartile range for continuous nonparametric variables, as mean and SD for continuous parametric variables, and count/total and percentages (\%) for categorical and dichotomous variables. Student T-test was used to analyze the continuous variables between the two studied groups and Chi-test for categorical and dichotomous variables. The level of statistical significance was $p<0.05$. Presentation of the statistical outcomes in the form of tables and graphs was performed using the "Microsoft Office Excel® 2007" program.

\section{Results}

Thirty patients were included in this study and divided into two groups; Study (Fibromyalgia) group and control group. Each group had fifteen patients. All the subjects in both groups were females. Age of the subjects ranged from 25 to 50 years among study group and ranged from 22 to 50 years among control group. Nine patients in fibromyalgia group complained of vertigo; seven of them complained of sense of imbalance and two patients complained of lightheadedness, while in control group all patients had not complained of vertigo. Audiometric evaluation was done in both groups and all patients had normal hearing. The vestibular assessment was done by bedside examinations and Videonystagmography (VNG). Regarding caloric test 
results, figure 1 shows that in the study group 5 patients $(33.3 \%)$ had unilateral vestibular weakness and 10 patients (66.7\%) had normal caloric test. In control group 3 patient (20\%) had unilateral vestibular weakness and 12 patients ( $80 \%$ ) had normal caloric test but there was no statistically significant difference between both groups regarding results of the caloric test $(p$-value $=0.6)$. Figure two shows the distribution of peripheral vestibular assessment (caloric, stepping and positional tests) results in the nine patients who had a complaint of vertigo in fibromyalgia group as follows: five patients had abnormal test results; two had unilateral caloric weakness (degrees of weakness were $30 \%$ and 38\%), abnormal stepping and positional tests, two had unilateral caloric weakness only (degrees of weakness were $21 \%$ and $27 \%$ ) and one patient had unilateral caloric weakness (degree of weakness was $29 \%$ ) and abnormal stepping test, while the rest of nine patients (four patients) who complained of vertigo had normal results. On the other hand, all six patient who had not complained of vertigo had normal caloric, stepping, and positional tests.

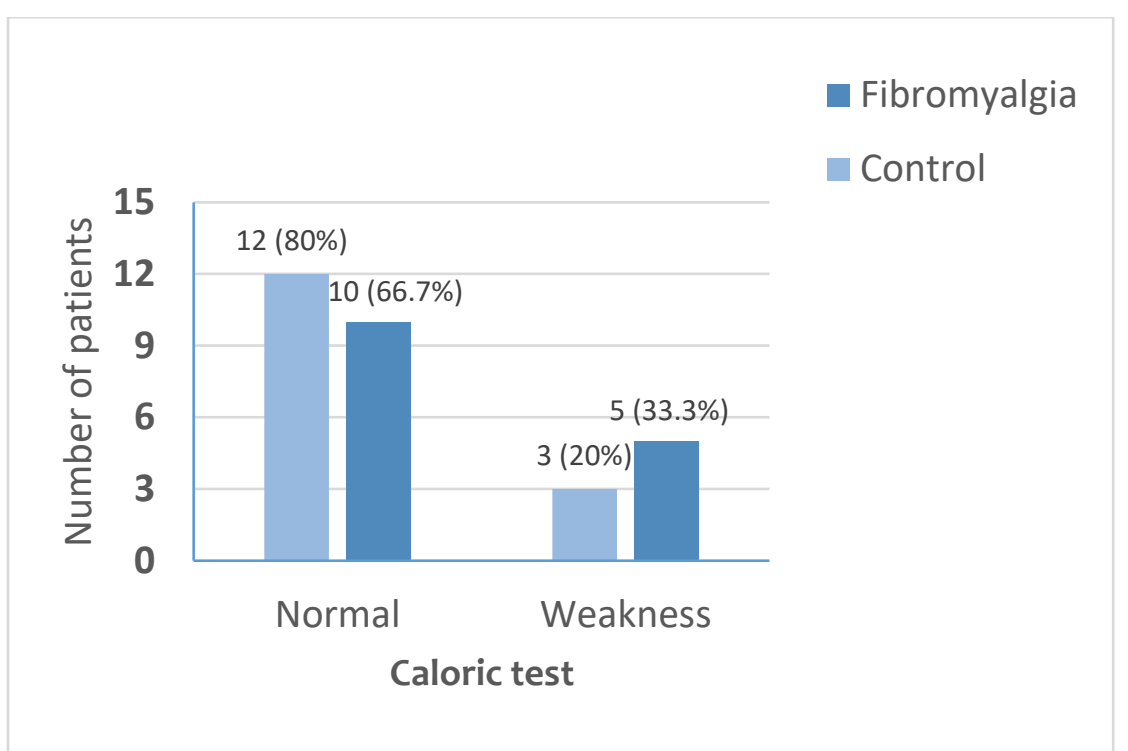

Figure 1: Bar chart showing results of Caloric test in control group and fibromyalgia group.

\section{Discussion}

Fibromyalgia is a non-inflammatory musculoskeletal chronic syndrome, whose etiology is unknown, characterized by a diffuse pain, increase in palpation sensitivity and such symptoms as tiredness, insomnia, anxiety, depression, cold intolerance and otologic complaints. The aim of this study to improve management of patient with fibromyalgia having dizziness. Thirty subjects were included in the current study and divided into two groups; Study (Fibromyalgia) group and control group. Each group had fifteen subjects. All subjects in both groups were females, as fibromyalgia more common in females. Age of the patients ranged from 25 to 50 years among study group and ranged from 22 to 50 years among control group that matched our inclusion criteria as age above 50 years may increase the occurrence of presbyastasis that may affect the results of the current study. In the present study, the complaint of vertigo was found to be present in $60 \%$ of patients (nine patients) in fibromyalgia group. This result was similar to those reported in Bayazit et al in 2002 ${ }^{(10)}$ that assessed the ear-related symptoms in 23 female patients with fibromyalgia. 


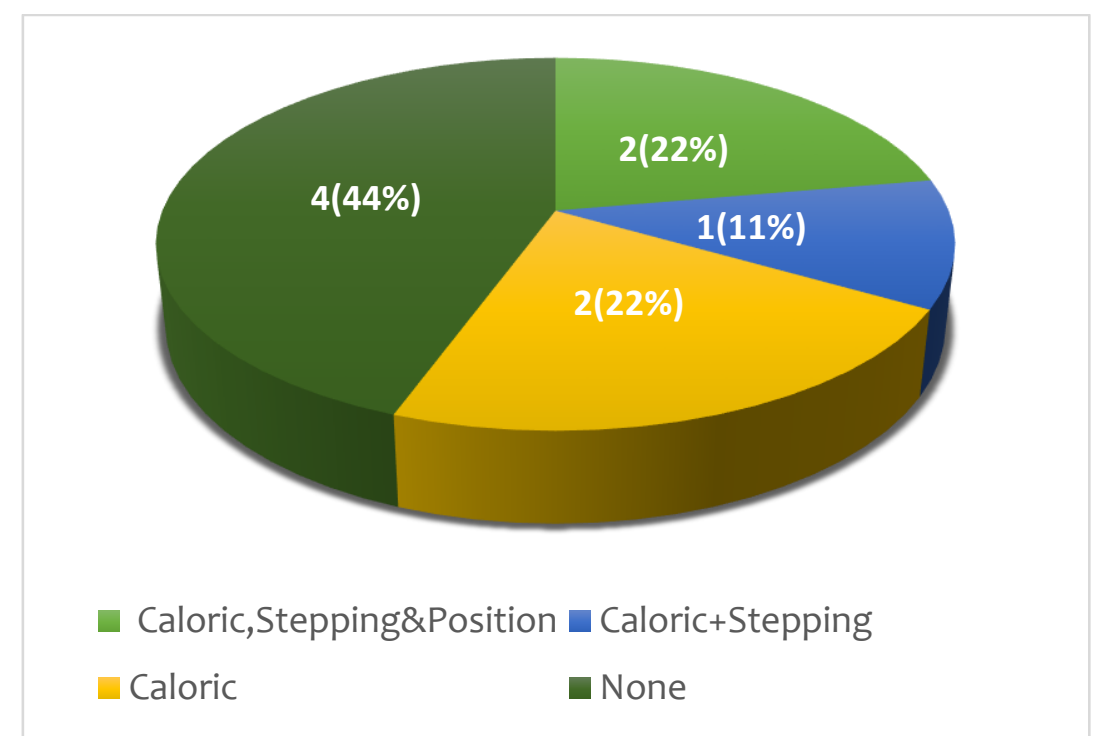

Figure 2: Pie chart showing peripheral vestibular assessment in patients with complaint. of vertigo in fibromyalgia group.

They found that $50 \%$ of the patients had either dizziness or vertigo. These observations agree with those previously observed by Watson et al in 2009(11) who also evaluated the frequency of various neurological signs and symptoms in both fibromyalgia patients and healthy control subjects. Each subject completed a physical examination by a neurologist as well as a questionnaire designed to assess neurologic symptoms over the previous three months. The researchers found that 30\% of fibromyalgia patients had vertigo compared to $1 \%$ of healthy control subjects only. Similarly, Zeigelboim BS et al in 2011(1) evaluated the vestibular behavior in fibromyalgia patients and the study was performed on 25 female patients diagnosed as fibromyalgia without middle ear pathologies. Otorhinolaryngological history in these patients showed dizziness in 21 patients (84\%) from 25 fibromyalgia female patients. In concordance with the findings of the current study, Koca $\mathrm{T}$ et al in $2018^{(12)}$ compared 44 patients with FM and 44 healthy volunteers. Vertigo and balance complaints were significantly higher in the FM group. All subunits (emotional, functional and physical) and total scores of dizziness handicap inventory were significantly higher in the FM group. In the current study, it was found that seven patients (78\%) in study group complained of sense of imbalance with long term complaint of vertigo (mean of 30 months) that might be related to musculoskeletal abnormalities as it was noticed that musculoskeletal abnormalities was significantly higher in fibromyalgia group. Also, it was noticed that the mean duration of the disease in patients who had complained of vertigo in fibromyalgia group was statistically significantly higher ( 4.5 years) compared with 1.5 years in patients who had not complained of vertigo in fibromyalgia group. Similarly, Zeigelboim BS et al in 2011 ${ }^{(1)}$ explained the dizziness in 21 patients from 25 fibromyalgia female patients by musculoskeletal abnormalities that are common in FM and they can impair the proprioception by causing an instability of sensation. The complaint of vertigo might also be related in some patients in fibromyalgia group to the usage of drugs such as analgesics and anti-depressants because they can cause dizziness and drowsiness as side effects (reported in $8 \%$ of all patients using these drugs) specially when used for longer 
duration (mean duration of usage in present study was 1.5 years) also, dizziness may occur with abrupt withdrawal from SSRI(13) which wasn't reported in the current study. A different explanation was introduced by Koca $T$ et al in $2018^{(12)}$ who found that vertigo and balance complaints were significantly higher in the FM group (84\%) and explained their results by high oxidative stress activity in fibromyalgia because increased active oxygen derivatives called oxidants (which are free radicals such as lipid peroxide, protein carbonyl and nitric oxide) can cause damage to hair cells and induce dysfunction of microvascular circulation in the peripheral vestibular system. In the present study audiometric evaluation was done in both groups and all patients had normal hearing, this finding showed that fibromyalgia does not affect cochlea, as found by Bayazit et al in $2002^{(10)}$ who found that the audiometry results of 23 patients with $F M$ were all normal. On the contrary to other studies, Kapusuz Gencer $Z$ et al in $2017^{(14)}$ conducted a case-control study, enrolling 35 patients with FM and 25 healthy volunteers as the control group. Although at low $(250-2000 \mathrm{~Hz})$ frequencies, the mean air and bone conduction threshold values between the two groups were not statistically significant $(p>0.05)$ and within normal range. Yet, at high frequencies (4000$8000 \mathrm{~Hz}$ ), air-bone conduction threshold values revealed hearing loss in the $F M$ group. The speech discrimination scores (SDS) of these patients were also diminished; however, these values did not reach statistical significance compared to control group's values ( $p>0.05$ ). Regarding tympanometric values, there was no statistically significant difference among the groups ( $p>0.05)$. The difference between the results of the two studies could be explained by increased age in FM group in that study (ranging up to 65 years with mean age of 48 years) which may be associated with presbycusis. In the present study, regarding bedside examinations; there was no spontaneous nystagmus, no gaze-evoked nystagmus, and no post head-shaking nystagmus in both groups. However stepping test results showed that in fibromyalgia group 3 patients (20\%) showed deviation in the stepping test while 2 subjects $(13.4 \%)$ showed deviation in control group with no statistically significant difference between both groups and that deviation in control group is unlikely to be explained by decompensation or imbalance of vestibular system as these subjects had normal other bedside and VNG test results and stepping test is less reliable screening tool for peripheral vestibular asymmetry as its sensitivity is (50\%) and specificity is $(60 \%) .{ }^{(15)}$. Moreover, Dix-hallpike test results showed normal test results among all subjects in both groups. In the current study V.N.G tests showed the same results as the office tests regarding spontaneous nystagmus and gaze-evoked nystagmus, also tracking test showed normal results in both groups. Moreover, Optokinetic test results showed decreased gain in 3 patients of $F M$ group (20\%), also saccade test results showed abnormal accuracy and latency in 3 patients of FM group (20\%) and these results might be explained by decreased reliability of those patients as these tests are highly affected by inattention of patients and these tests have poor sensitivity in identifying central vestibular lesion giving false positive results ${ }^{(16)}$. Positional Test showed abnormal results in two patients of fibromyalgia group (13.3\%), both had significant nystagmus. In control group one patient (6.7\%) had significant nystagmus with no statistically significant difference between both groups. Those abnormal results are unlikely explained by uncompensated peripheral vestibular lesion as positional test has moderate sensitivity and specificity ${ }^{(17)}$. Zeigelboim BS et al in 2011 ${ }^{(1)}$ evaluated the 
vestibular behavior in one group of $25 \mathrm{fi}$ bromyalgia patients by the following tests integrating the vestibular exam: positional/positioning, pendular track and optokinetic nystagmus did not show alterations. Caloric Test results showed that in the study group 5 patients (33.3\%) had unilateral vestibular weakness, while in control group 3 patient (20\%) had unilateral vestibular weakness, there was no statistically significant difference between both groups ( $p$-value $=0.6$ ). In contrast to results of the current study Pérez-deHeredia-Torres M et al in $2017^{(18)}$ compared the sensory organization test of balance between women with fibromyalgia (FM) and healthy women by (Posturo-graphy) and the results showed that women with FM displayed significantly lower values in all SOT conditions (vestibular, visual and somatosensory) than healthy women and scores of conditions 4-6 were significantly lower than those for conditions 1-3. The difference between results of the current study and results of that study is that caloric test assesses vestibulo-ocular function, while sensory organization tests assess vestibulo-spinal function and many patients with fibromyalgia had musculoskeletal abnormalities, so they could have worse balance scores at posturography. The distribution of peripheral vestibular assessment (caloric, stepping, and positional tests) in fibromyalgia group showed that five patients had unilateral caloric weakness and two of them only had abnormal stepping and positional tests and those two patients are likely to have peripheral vestibular dysfunction as each test alone has its limited specificity and sensitivity while using a combination of several clinical tests got the most reliable vestibular assessment. In the current study regarding bed side examinations and VNG results; it was concluded that there was a trend towards positive results and increase in ves- tibular weakness in fibromyalgia group compared to control group but, without statistically significant difference between both groups thus, fibromyalgia is unlikely to be a cause of peripheral vestibular dysfunction however, it might be a cause of complaint of vertigo through musculoskeletal abnormalities, longer duration of the disease and usage of medications as analgesics and antidepressants. From all the above, we accept the null hypothesis that there is no significance difference in peripheral vestibular dysfunction between two groups. Bedside tests and caloric test had some limitations such as caloric testing has low sensitivity and specificity, and assesses only the function of the horizontal semicircular canal(19) also, caloric response stimulates very low frequencies ( 0.002 to 0.004 ) which are well below the physiological range of the VOR, so patients with normal caloric test results may have vestibular weakness at higher frequencies and may need more investgations such as Video-head impulse test ( $\mathrm{VHIT}$ ) which is a more physiological stimulus testing higher frequencies $(>1 \mathrm{~Hz})$ and can detect abnormalities in all six semicircular canals ${ }^{(20)}$, also Computerized Dynamic Posturography (CDP) can assess vestibulo-spinal function (Visual, vestibular, somatosensory) and vestibular-Evoked-Myogenic-Potential (VE MP) that asses otolith organs. Finally, the current study presents some limitations; first, Scarcity of cases of fibromyalgia visitng the hospital and second, not all vestibular tests were done as fibromyalgia patients can't tolerate having all vestibular tests together as they will take very long time.

\section{Conclusion}

Fibromyalgia is unlikely to be a cause of peripheral vestibular dysfunction. Thus, no vestibular testing is needed for such patients. 


\section{References}

1. Zeigelboim BS, Moreira DN. Vestibular findings in fibromyalgia patients. Arquivos Internacionais de Otorrinolaringologia. 2011; 15(03):283-9.

2. Staud R. Autonomic dysfunction in fibromyalgia syndrome: postural orthostatic tachycardia. Current rheumatology reports. 2008;10(6):463.

3. Baloh RW. Vestibular neuritis. New England Journal of Medicine. 2003;348(11):1027-32.

4. Lee H, Sohn S-I, Cho Y-W, Lee S-R, Ahn B-H, Park B-R, et al. Cerebellar infarction presenting isolated vertigo frequency and vascular topographical patterns. Neurology. 2006;67 (7):117883.

5. Huh YE, Kim JS. Patterns of spontaneous and head-shaking nystagmus in cerebellar infarction: imaging correlations. Brain. 2011;134 (12):3662-71.

6. Huh Y-E, Kim J-S. Bedside evaluation of dizzy patients. Journal of Clinical Neurology. 2013;9(4):203-13.

7. Zapala DA. Down-beating nystagmus in anterior canal benign paroxysmal positional vertigo. Journal of the American Academy of Audiology. 2008;19(3):257-66.

8. Fife T, Tusa R, Furman J, Zee D, Frohman E, Baloh R, et al. Assessment: Vestibular testing techniques in adults and children Report of the Therapeutics and Technology Assessment Subcommittee of the American Academy of Neurology. Neurology. 2000;55(10):1431-41.

9. Schoepflin JR. Back to basics: Speech audiometry. Adelphi University Retrieved on. 2012;23(12):2014.

10. Bayazıt YA, Gürsoy S, Özer E, Karakurum G, Madenci E. Neurotologic manifestations of the fibromyalgia syndrome. Journal of the neurological sciences. 2002;196(1-2):77-80.

11. Watson NF, Buchwald D, Goldberg J, Noonan C, Ellenbogen RG. Neurologic signs and symptoms in fibromyalgia.
Arthritis \& Rheumatism. 2009; 60(9): 2839-44.

12. Koca T, Seyithanoğlu M, Sagiroglu $S$, Berk E, Dağlı $H$. Frequency of audiological complaints in patients with fibromyalgia syndrome and its relationship with oxidative stress. Nigerian journal of clinical practice. 2018;21(10):1271-7.

13. Smith PF, Darlington CL. A possible explanation for dizziness following SSRI discontinuation. Acta otolaryngologica. 2010;130(9):981-3.

14. Gencer ZK, BALBALOĞLU Ö, ÖZKIRIŞ $M$, Saydam L. Does fibromyalgia have an effect on hearing loss in women? Turkish journal of medical sciences. 2017;47(6):1699-702.

15. Honaker JA, Boismier TE, Shepard NP, Shepard NT. Fukuda stepping test: sensitivity and specificity. Journal of the American Academy of Audiology. 2009;20(5):311-4.

16. Shepard NT, Telian SA. Practical management of the balance disorder patient: Singular; 1996.

17. Mohamed ES. Predictors of central vestibular disorders from videonystagmography tests. The Egyptian Journal of Otolaryngology. 2016; 32(3):202.

18. Pérez-de-Heredia-Torres $M$, HuertasHoyas E, Martínez-Piédrola R, PalaciosCeña D, Alegre-Ayala J, SantamaríaVázquez $M$, et al. Balance deficiencies in women with fibromyalgia assessed using computerised dynamic posturography: a cross-sectional study in Spain. BMJ Open. 2017;7(7):e016239.

19. Aminoff MJ. Aminoff's Electrodiagnosis in Clinical Neurology: Expert Consult-Online and Print: Elsevier Health Sciences; 2012.

20. Bell SL, Barker F, Heselton H, MacKenzie E, Dewhurst D, Sanderson A. A study of the relationship between the video head impulse test and air calorics. European Archives of OtoRhino-Laryngology. 2015;272(5):128794. 